\title{
A note from the Editor-in-Chief
}

Nava Segev

Editor-in-Chief; Cellular Logistics; and Department of Biological Sciences; University of Illinois at Chicago; Chicago, IL USA

We are starting the second year of Cellular Logistics with a first issue that includes a research paper and a number of reviews and addenda on diverse topics in the field.

We are in the midst of applying for inclusion in Medline, which among other benefits for the journal would decrease the time between acceptance of submissions and their appearance on PubMed.

Our goals for this year are to increase the scope of the journal and continue to provide a stage for reasoned debates on scientific disputes. Rick Kahn will lead this latter effort. While we received positive feedback to the CellLogBlog content, it was also deemed hard to find and use. We decided to try another approach. The articles intended to highlight and discuss controversial topics will become a regular feature within the journal, with the intent of getting input from the readers. To facilitate discussions among scientists, we added a "Comments" link below every paper on the website. To participate in the discussion, you need to create a free account that includes your email.

Nava Segev

Editor-in-Chief

Cellular Logistics 